\title{
Time-domain analysis of attosecond pulse generation in an $x$-ray free-electron laser
}

\author{
Panagiotis Baxevanis, Joseph Duris, Zhirong Huang, and Agostino Marinelli \\ SLAC National Accelerator Laboratory, Menlo Park, California 94025, USA
}

(Received 1 May 2018; revised manuscript received 18 October 2018; published 8 November 2018)

\begin{abstract}
The method of enhanced self-amplified spontaneous emission (eSASE) is one of the strongest candidates for the generation of sub-femtosecond x-ray pulses in a free-electron laser (FEL). The optimization of an eSASE experiment involves many independent parameters, which makes the exploration of the parameter space with thee-dimensional (3D) simulations computationally intensive. Therefore, a robust theoretical analysis of this problem is extremely desirable. We provide a self-consistent, analytical treatment of such a configuration using a one-dimensional (1D), time-dependent FEL model that includes the key effects of electron beam chirp and undulator taper. Verified via comparison with numerical simulation, our formalism is utilized in parameter studies that seek to determine the optimum setup of the FEL.
\end{abstract}

DOI: 10.1103/PhysRevAccelBeams.21.110702

\section{INTRODUCTION}

The generation of ultrashort $\mathrm{x}$-ray pulses is one of the main objectives of research into advanced operation modes for a modern FEL facility [1-4]. One of the most promising schemes for generating sub-fs x-rays in a high-gain FEL is eSASE (enhanced self-amplified spontaneous emission) [5]. This technique involves the interaction of an electron beam with an optical laser pulse in the presence of a short wiggler, prior to the beam being sent into a conventional undulator. Apart from providing an attractive mechanism for generating X-ray pulses in the attosecond range, this method also results in a significant improvement in the performance of the FEL. In common with some other schemes for generating ultrashort x-rays [6], the eSASE process essentially relies on an intense manipulation of the longitudinal phase space of the electrons, after which the e-beam is strongly chirped and typically has to travel through a tapered undulator in order to achieve lasing with the required properties. Thus, from an FEL physics point of view, the key features of eSASE are beam chirp and undulator taper, as well as the finite-pulse effects associated with a short (fs-level) electron bunch. In the context of FEL theory [7], these topics are typically studied in the onedimensional (1D) regime, where effects such as radiation diffraction, emittance and focusing can be neglected. Even in this approximation, however, a comprehensive analytical treatment of this important subject is lacking. In particular,

Published by the American Physical Society under the terms of the Creative Commons Attribution 4.0 International license. Further distribution of this work must maintain attribution to the author(s) and the published article's title, journal citation, and DOI. most of the theoretical literature deals with short-pulse effects [8,9] or beam chirp alone [10-12], without combining these topics or including the effect of taper in the analytical calculations.

In this paper, we provide a generalized, self-consistent, time-domain analysis of an FEL configuration that utilizes a short, chirped e-beam and a tapered undulator. Aiming to model the FEL portion of an eSASE-based scheme, we use a simplified 1D formalism that also includes the key effects of startup from shot noise and radiation slippage. Our treatment is based on the linearized Maxwell-Vlasov equations of the FEL and therefore is valid for that stage of the interaction prior to the onset of saturation. The main objective is to determine the basic properties of the generated radiation pulse (such as the peak radiation power and the radiation pulse length) and to investigate how these depend on the various parameters of the system. In this way, we hope to obtain a more thorough understanding of the physics behind the experimental technique.

This paper is organized as follows: Section II contains the bulk of the theoretical development. Starting from the time-dependent Maxwell-Vlasov equations, a single evolution equation is obtained for the amplitude of the FEL radiation, assuming startup from noise. Next, the evolution equation is solved through a Laplace transform technique, leading to a description of the radiation field in terms of a Green's function. This, in turn, enables us to calculate various key figures of merit for the FEL in the latter stage of the exponential gain regime. This capability is exploited in Sec. III, which presents the results of a numerical study based on our method. After checking its validity through comparison with appropriate FEL simulation data, our semianalytical formalism is utilized in parameter studies that seek to determine the optimum setup of an 
eSASE-based FEL. The main results of this paper are summarized in Sec. IV.

\section{THEORY}

\section{A. Maxwell-Vlasov analysis}

The starting point of our treatment is the equations of motion for a single electron in the combined field of the FEL radiation and the undulator. In the context of our onedimensional analysis, the appropriate phase space variables are $(\theta, \eta)$, where $\theta=k_{u} z+k_{r}(z-c \bar{t})$ is the ponderomotive phase and $\eta=\gamma / \gamma_{0}-1$ is the relative energy deviation. Here, $k_{u}=2 \pi / \lambda_{u}, k_{r}=2 \pi / \lambda_{r}, \bar{t}$ is the arrival time averaged over the fast wiggle motion and $\gamma_{0}$ is the average relativistic factor of the electrons. The undulator period $\lambda_{u}$ and the resonant wavelength $\lambda_{r}$ are related through the wellknown resonance condition $\lambda_{r}=\lambda_{u}\left(1+K_{0}^{2} / 2\right) /\left(2 \gamma_{0}^{2}\right)$, where $K_{0}$ is the dimensionless (initial) undulator parameter. For future reference, it is also worth noting that the phase $\theta$ is related to the longitudinal position within the electron bunch $z_{b}$ via $\theta=k_{r} z_{b}$. In fact, we have $0 \leq \theta \leq \theta_{b}=k_{r} l_{b}$ ( $l_{b}$ is the bunch length), where the upper/lower limits respectively represent the head/tail of the bunch. The electric field of the radiation, which is assumed to be linearly polarized along the $x$-axis, is given by $E_{\text {rad }}=$ $E_{x}=A(\theta, z) \exp \left(i k_{r}(z-c t)\right)+$ c.c., where $A(\theta, z)$ is a slowly varying amplitude and c.c. stands for complex conjugate. The fact that the amplitude $A$ is $\theta$-dependent is a principal feature of the time-domain analysis, facilitating the description of a short radiation pulse. Moreover, the meaning of the term "slowly varying" is to be understood in the following way: the characteristic length in $z$ for a significant variation of $A$ (gain length) is much larger than $\lambda_{u}$, while the corresponding length in terms of the internal bunch position $\theta / k_{r}$ (cooperation length) is much larger than $\lambda_{r}$ (the actual length scales will be quantified later on). As far as the undulator is concerned, we assume that the undulator parameter $K$ is tapered according to the relation $K=K_{0}(1+\varepsilon z)$, where $\varepsilon$ is a constant linear taper gradient. The single-particle dynamics of the electrons (after averaging over many undulator periods) is determined by the well-known pendulum equations, suitably modified for the case of a tapered undulator. These are [13]

$$
\frac{d \theta}{d z}=2 k_{u} \eta+a_{1} z
$$

and

$$
\frac{d \eta}{d z}=\zeta_{1} A(\theta, z) \exp (i \theta)+\text { c.c. }
$$

where $a_{1}=-2 k_{u} K_{0}^{2} \varepsilon /\left(2+K_{0}^{2}\right)$ and $\zeta_{1}=e K_{0}[J J] /\left(2 \gamma_{0}^{2} m c^{2}\right)$. In the definitions given above $e$ and $m$ are the electron charge and mass while $[J J]$ is an averaging factor given by $[J J]=J_{0}\left(Q_{0}\right)-J_{1}\left(Q_{0}\right)$, where $Q_{0}=K_{0}^{2} /\left(4+2 K_{0}^{2}\right)$ and $J_{0}, J_{1}$ are Bessel functions.
In order to describe the interaction between the electron beam and the radiation in a self-consistent fashion, we introduce a distribution function $f(\theta, \eta, z)$ that is a measure of the beam phase space density. The distribution function $f$ is constant along an individual electron trajectory, so that $\partial f / \partial z+(\partial f / \partial \theta)(d \theta / d z)+(\partial f / \partial \eta)(d \eta / d z)=0$. This evolution equation for $f$ (i.e., the Vlasov equation) is, generally speaking, nonlinear in nature. However, in the socalled linear regime of the FEL interaction (comprising the initial lethargy stage and the subsequent exponential gain region) the analysis can be considerably simplified by following a perturbation approach. In particular, we assume that the distribution function can be written as $f=f_{0}+f_{1}$, where $f_{0}$ is a background (or unperturbed) part and $f_{1}$ is a small perturbation due to the FEL effect (so that $\left|f_{1}\right| \ll f_{0}$ ). $\mathrm{Up}$ to the beginning of the saturation region, this is a reasonably good approximation. Furthermore, we can express $f_{1}$ as $f_{1}=F(\theta, \eta, z) \exp (i \theta)+$ c.c., where the density amplitude $F$-like the field amplitude $A$-is a slowly varying function of $\theta$ and $z$. This decomposition is justified by the fact that the characteristic length for the microbunching is the radiation length $\lambda_{r}$, which is much shorter than the cooperation length. In the context of the abovementioned approximation, the phase space evolution of the electron beam is described by a linearized version of the Vlasov equation [9], namely

$$
\frac{\partial \bar{F}}{\partial z}+\left(2 k_{u} \eta+a_{1} z\right) \frac{\partial \bar{F}}{\partial \theta}=-\zeta_{1} A \exp (i \theta) \frac{\partial f_{0}}{\partial \eta},
$$

where we have introduced the complex quantity $\bar{F}=$ $F \exp (i \theta)$ (so that $f_{1}=\bar{F}+$ c.c.) and the unperturbed distribution $f_{0}$ satisfies the relation

$$
\frac{\partial f_{0}}{\partial z}+\left(2 k_{u} \eta+a_{1} z\right) \frac{\partial f_{0}}{\partial \theta}=0 .
$$

To complete our description of the system, we need a relation that quantifies the change in the radiation field in response to the currents associated with the electron beam. In general, this is facilitated by a driven, paraxial wave-type equation, which, in our case, takes the form [9]

$$
\frac{\partial A}{\partial z}+k_{u} \frac{\partial A}{\partial \theta}=-\zeta_{2} n_{0} \exp (-i \theta) \int d \eta \bar{F} .
$$

Here, the new coupling coefficient $\zeta_{2}$ is given by $\zeta_{2}=$ $e K_{0}[J J] /\left(4 \varepsilon_{0} \gamma_{0} \Sigma\right)$, where $\varepsilon_{0}$ is the vacuum permittivity and $\Sigma$ is a cross-sectional area. For a round beam with a Gaussian transverse profile, $\Sigma$ is equal to $2 \pi \sigma_{x}^{2}$, where $\sigma_{x}$ is the transverse rms beam size. Moreover, $n_{0}$ is the maximum value of the electron line density (i.e., the maximum number of electrons per unit length along the bunch).

An analytical solution to the linearized Vlasov equation [Eq. (3)] can be obtained using the method of integration 
along the unperturbed trajectories [7]. Omitting the details, the end result of this procedure is the relation

$$
\begin{aligned}
\bar{F} \approx & \bar{F}\left(\theta-2 k_{u} \eta z-g(z), \eta, 0\right)-\zeta_{1} \frac{\partial f_{0}}{\partial \eta} \int_{0}^{z} d s A(\theta, s) \\
& \times \exp \left(i \theta+2 i k_{u} \eta(s-z)+i\{g(s)-g(z)\}\right),
\end{aligned}
$$

where $g(z)=\int_{0}^{z} a_{1} t d t=a_{1} z^{2} / 2$. As far as the background distribution is concerned, the same analytical method yields a general solution that can be written as $f_{0}=\chi\left(\theta_{1}\right) V\left(\eta_{1}\right)$. In this relation, $\theta_{1}=\theta-2 k_{u} \eta z-g(z)$ and $\eta_{1}=$ $\eta+\mu\left(\theta_{1}-\theta_{m}\right)+Y\left(\theta_{1}\right)$, where $\chi(\theta), V(\eta)$ and $Y(\theta)$ are arbitrary functions, $\mu$ is a constant and $\theta_{m}=\theta_{b} / 2$. Assuming that $\theta \gg 2 k_{u} \eta z, g(z)$, we have $\theta_{1} \approx \theta$ and the $z$-dependent character of $f_{0}$ can be neglected, yielding $f_{0} \approx \chi(\theta) V\left(\eta+\mu\left(\theta-\theta_{m}\right)+Y(\theta)\right)$. The validity of this important approximation will be verified in due course.

From a physical point of view, the meaning of the various functions and parameters associated with $f_{0}$ is as follows: $\chi(\theta)$ is a dimensionless quantity that represents the scaled current profile of the electron bunch. In particular, we have $I(\theta)=I_{p} \chi(\theta)$ for $0 \leq \theta \leq \theta_{b}$-where $I_{p}=n_{0} e c$ is the peak current-and $\chi(0)=\chi\left(\theta_{b}\right)=0$. The function $V(\eta)$ represents the uncorrelated energy spread profile of the beam and satisfies the relation $\int d \eta V(\eta)=1$. In this paper, we neglect uncorrelated energy spread effects so we may assume that $V(\eta)=\delta(\eta)$ from now on. We do, however, take into account the correlated energy spread due to the chirp of the electron beam, which is modeled by $\eta=-\mu\left(\theta-\theta_{m}\right)-Y(\theta)$. Thus, $\mu$ is a linear chirp coefficient and $-Y(\theta)$ represents the contribution of a possible nonlinear chirp component.

Substituting Eq. (6) into the right-hand side (rhs) of Eq. (5) and making use of the expression for $f_{0}$ leads to a single, self-consistent equation for the radiation amplitude $A(\theta, z)$. In this study, we are only concerned with the case of SASE (self-amplified spontaneous emission), which corresponds to startup from shot noise. As a result, the process of deriving the required amplitude equation also involves making use of the representation $\bar{F}(\theta, \eta, 0) \rightarrow$ $\left(k_{r} / n_{0}\right) \sum_{j} \delta\left(\theta-\theta_{j}\right) \delta\left(\eta-\eta_{j}\right)$, where $\theta_{j}$ are the random initial phases of the electrons-i.e., the phases at $z=0$ and $\eta_{j}$ are the corresponding energies (the summation over the discrete index $j$ extends over all electrons of the bunch). The final result of this manipulation is

$$
\begin{aligned}
\left(\frac{\partial}{\partial z}\right. & \left.-i a_{1} z+k_{u} \frac{\partial}{\partial \theta}\right) \tilde{A}(\theta, z) \\
= & -\zeta_{2} k_{r} \sum_{j} \exp \left(-i \theta_{j}\right) \\
& \times \exp \left(2 i k_{u} z\left\{\mu\left(\theta_{j}-\theta_{m}\right)+Y\left(\theta_{j}\right)\right\}\right) \delta\left(\theta-\theta_{j}\right)-8 i \rho^{3} k_{u}^{3} \\
& \times \chi(\theta) \int_{0}^{z} d s \tilde{A}(\theta, s)(s-z) \exp \left[-2 i k_{u}\left\{\mu\left(\theta-\theta_{m}\right)\right.\right. \\
& +Y(\theta)\}(s-z)],
\end{aligned}
$$

where $\tilde{A}(\theta, z)=A(\theta, z) \exp \left(i a_{1} z^{2} / 2\right)$ and $\rho$ is the FEL (or Pierce) parameter, introduced here through the relation $\zeta_{1} n_{0} \zeta_{2}=4 k_{u}^{2} \rho^{3}$.

\section{B. Green's function}

To solve an integral-differential equation like Eq. (7), we typically make use of a Laplace transform technique. In our case, we introduce an auxiliary amplitude quantity $\hat{A}(\theta, \lambda)$ via

$$
\hat{A}(\theta, \lambda)=\int_{0}^{\infty} d z \exp \left(2 i k_{u} \lambda z\right) \tilde{A}(\theta, z)
$$

and substitute Eq. (8) back into Eq. (7). This results in a partial differential equation for $\hat{A}(\theta, \lambda)$, namely

$$
\begin{aligned}
& \frac{\partial \hat{A}(\theta, \lambda)}{\partial \theta}-\bar{a}_{1} \frac{\partial \hat{A}(\theta, \lambda)}{\partial \lambda}-2 i \lambda \hat{A}(\theta, \lambda)+\frac{2 i \rho^{3} \chi(\theta) \hat{A}(\theta, \lambda)}{\left(\mu\left(\theta-\theta_{m}\right)+Y(\theta)+\lambda\right)^{2}} \\
& =\frac{\tilde{A}(\theta, 0)}{k_{u}}+\frac{\zeta_{2} k_{r}}{2 k_{u}^{2}} \sum_{j} \frac{\exp \left(-i \theta_{j}\right) \delta\left(\theta-\theta_{j}\right)}{i \lambda+i \mu\left(\theta_{j}-\theta_{m}\right)+i Y\left(\theta_{j}\right)}
\end{aligned}
$$

where $\bar{a}_{1}=a_{1} /\left(2 k_{u}^{2}\right)$. For the SASE case under consideration, the initial electric field amplitude is zero so $\tilde{A}(\theta, 0)=0$. Taking this into account, we find that the solution to Eq. (9) can be written as

$$
\begin{aligned}
\hat{A}(\theta, \lambda)= & \frac{\zeta_{2} k_{r}}{2 i k_{u}^{2}} \int_{-\infty}^{\theta} d \theta^{\prime} \exp \left[\Delta \bar{Q}\left(\theta, \theta^{\prime}, \lambda\right)\right] \\
& \times \sum_{j} \frac{\exp \left(-i \theta_{j}\right) \delta\left(\theta^{\prime}-\theta_{j}\right)}{\lambda+\bar{a}_{1} \theta+\mu\left(\theta_{j}-\theta_{m}\right)+Y\left(\theta_{j}\right)-\bar{a}_{1} \theta_{j}},
\end{aligned}
$$

where

$$
\begin{aligned}
\Delta \bar{Q}\left(\theta, \theta^{\prime}, \lambda\right)= & -2 i \lambda\left(\theta^{\prime}-\theta\right)+i \bar{a}_{1}\left(\theta^{\prime}-\theta\right)^{2} \\
& +2 i \rho^{3} \int_{\theta}^{\theta^{\prime}} \frac{d \xi \chi(\xi)}{\left[\lambda+\bar{a}_{1} \theta+\mu\left(\xi-\theta_{m}\right)+Y(\xi)-\bar{a}_{1} \xi\right]^{2}}
\end{aligned}
$$

The inverse Laplace transform is given by

$$
\tilde{A}(\theta, z)=\frac{k_{u}}{\pi} \int_{-\infty+i y}^{+\infty+i y} d \lambda \exp \left(-2 i k_{u} \lambda z\right) \hat{A}(\theta, \lambda),
$$

where the path of the integration is parallel to the real axis and above all singularities of the integrand. Combining Eqs. (10), (11), and (12), we obtain the following expression for the original field amplitude $A(\theta, z)=$ $\tilde{A}(\theta, z) \exp \left(-i a_{1} z^{2} / 2\right)$ :

$$
A(\theta, z)=-\frac{\zeta_{2} k_{r}}{k_{u}} \sum_{j} \exp \left(-i \theta_{j}\right) G\left(\theta, \theta_{j}, z\right),
$$


where $G\left(\theta, \theta_{j}, z\right)$ is the Green's function given by

$$
\begin{aligned}
G\left(\theta, \theta_{j}, z\right)= & -\frac{\exp \left[i \Psi_{2}\left(\theta, \theta_{j}, z\right)\right]}{2 \pi i} \\
& \times \int_{-\infty+i y}^{+\infty+i y} \frac{d \lambda}{\lambda} \exp \left(-2 i \lambda\left[k_{u} z-\left(\theta-\theta_{j}\right)\right]-2 i \rho^{3}\right. \\
& \left.\times \int_{\theta_{j}}^{\theta} \frac{d \xi \chi(\xi)}{\left[\lambda+\left(\mu-\bar{a}_{1}\right)\left(\xi-\theta_{j}\right)+Y(\xi)-Y\left(\theta_{j}\right)\right]^{2}}\right)
\end{aligned}
$$

and the phase term $\Psi_{2}$ is given by

$$
\begin{aligned}
\Psi_{2}\left(\theta, \theta_{j}, z\right)= & -\bar{a}_{1}\left[\theta-k_{u} z+\theta_{j}\right]\left(\theta-\theta_{j}-k_{u} z\right) \\
& +2\left\{\mu \theta_{m}-\left(\mu-\bar{a}_{1}\right) \theta_{j}-Y\left(\theta_{j}\right)\right\}\left(\theta-\theta_{j}-k_{u} z\right) .
\end{aligned}
$$

It is worth noting that $\left(\partial / \partial z+k_{u} \partial / \partial \theta\right) \Psi_{2}\left(\theta, \theta_{j}, z\right)=0$ since $\Psi_{2}$ depends on $\theta$ and $z$ through the combination $\theta-k_{u} z$. The field Green's function $G\left(\theta, \theta_{j}, z\right)$ is the principal result of our analysis. Multiplied by the factor $\exp \left(-i \theta_{j}\right)$, it represents the contribution of the $j$ th electron to the radiation amplitude. Moreover, it is only nonzero for $0 \leq \theta-\theta_{j} \leq k_{u} z$, as one could infer from causality arguments. In the case of zero nonlinear chirp $(Y(\theta)=0)$ and zero undulator taper $\left(\bar{a}_{1}=0\right)$, Eq. (14) is equivalent to the result of [10] regarding the Green's function for a pure linear chirp. Another important remark concerns the case with $\mu, \bar{a}_{1} \neq 0$ and $\mu=\bar{a}_{1}$ (still in the absence of nonlinear chirp), for which $|G|$ is identical to what it would be when $\mu=\bar{a}_{1}=0$. Since-as will be shown below-the absolute value of $G$ determines the power profile of the radiation, this observation corresponds to the well-known fact that the power-degrading effects of linear beam chirp can be removed by the appropriate linear taper [14]. In fact, the condition $\mu=\bar{a}_{1}$ readily reproduces the chirp-taper matching condition postulated (but not proven) in Ref. [6].

Knowledge of the Green's function allows us to determine the basic properties of the radiation and the electron beam in the linear regime. To begin with, we consider the shot-averaged radiation power $P_{r}(\theta, z)$ defined by

$$
P_{r}(\theta, z)=2 \Sigma \varepsilon_{0} c\left\langle|A|^{2}\right\rangle_{\text {shot }},
$$

where the symbol \langle\rangle$_{\text {shot }}$ denotes average over many different ensembles of random initial phases $\theta_{j}$. In view of Eq. (13), we obtain the result

$$
\begin{aligned}
|A|^{2}= & \left(\frac{\zeta_{2} k_{r}}{k_{u}}\right)^{2}\left[\sum_{j}\left|G\left(\theta, \theta_{j}, z\right)\right|^{2}\right. \\
& \left.+\sum_{k \neq j} \exp \left(-i\left(\theta_{j}-\theta_{k}\right)\right) G\left(\theta, \theta_{j}, z\right) G^{*}\left(\theta, \theta_{k}, z\right)\right] .
\end{aligned}
$$

In the absence of density gradient-related coherent effects $[8,9]$, the second term in the equation given above can be disregarded. This is a reasonable assumption for $\lambda_{r} \ll l_{b}$. The remaining sum can be converted to an integral via the substitution $\sum_{j} \rightarrow\left(n_{0} / k_{r}\right) \int d \theta_{j} \chi\left(\theta_{j}\right)$. The end result of this manipulation is the relation

$$
P_{r}(\theta, z)=4 \gamma_{0} m c^{3} k_{r} \rho^{3} \int d \theta_{j} \chi\left(\theta_{j}\right)\left|G\left(\theta, \theta_{j}, z\right)\right|^{2}
$$

for the radiation power. The above equation can be used to obtain the shot-averaged power profile of the FEL radiation.

A similar result can be obtained for the local bunching factor $b(\theta, z)=\left|\left\langle\exp \left(-i \theta_{l}\right)\right\rangle_{\Delta}\right|$, which expresses the degree to which the electron beam is bunched due to the FEL effect. Here, the $\Delta$-average takes into account only those electron phases $\theta_{l}$ for which $\theta_{l} \in(\theta, \theta+2 \pi)$. In terms of the beam distribution function $f$, we have

$\left\langle\exp \left(-i \theta_{l}\right)\right\rangle_{\Delta}=\frac{\int_{\theta}^{\theta+2 \pi} d \theta^{\prime} \int d \eta f\left(\theta^{\prime}, \eta, z\right) \exp \left(-i \theta^{\prime}\right)}{\int_{\theta}^{\theta+2 \pi} d \theta^{\prime} \int d \eta f\left(\theta^{\prime}, \eta, z\right)}$.

Recalling that $f$ is given by $f=f_{0}+f_{1}$ (with $\left|f_{1}\right| \ll f_{0}$, $f_{1}=\bar{F}+$ c.c. and $\bar{F}=F \exp (i \theta)$ ), the denominator becomes $\int_{\theta}^{\theta+2 \pi} d \theta^{\prime} \int d \eta f_{0}=2 \pi \chi(\theta)$ while the numerator is equal to $\int_{\theta}^{\theta+2 \pi} d \theta^{\prime} \int d \eta f_{1} \exp \left(-i \theta^{\prime}\right)=2 \pi \exp (-i \theta) \int d \eta \bar{F}$, where we have also made use of the fact that $f_{0} \approx \chi(\theta) V(\eta)$ and $\int d \eta V(\eta)=1$. In view of the above observations and Eq. (5), Eq. (18) becomes

$$
b(\theta, z)=\frac{1}{\chi(\theta)}\left|\int d \eta \bar{F}\right|=\frac{1}{\zeta_{2} n_{0} \chi(\theta)}\left|\frac{\partial A}{\partial z}+k_{u} \frac{\partial A}{\partial \theta}\right|,
$$

which, when combined with Eq. (13), leads to

$$
b(\theta, z)=\frac{k_{r}}{\chi(\theta) n_{0} k_{u}}\left|\sum_{j} \exp \left(-i \theta_{j}\right) G_{b}\left(\theta, \theta_{j}, z\right)\right|,
$$

where $G_{b}\left(\theta, \theta_{j}, z\right) \equiv\left(\partial / \partial z+k_{u} \partial / \partial \theta\right) G\left(\theta, \theta_{j}, z\right)$ is a derivative Green's function. An inspection of Eq. (14) shows that $G$ is a function of $\theta-k_{u} z$ for $\theta>\theta_{b}$, in which case $G_{b}=0$. Thus, unlike $G$, the derivative (or bunching) Green's function $G_{b}$ is nonzero only within the limits of the electron bunch. A procedure entirely analogous to the one we followed for the radiation power yields a result for the shot-averaged bunching factor, namely

$$
\left\langle b^{2}\right\rangle_{\text {shot }}=\frac{k_{r}}{[\chi(\theta)]^{2} n_{0} k_{u}^{2}} \int d \theta_{j} \chi\left(\theta_{j}\right)\left|G_{b}\left(\theta, \theta_{j}, z\right)\right|^{2} .
$$

Though a product of a linearized analysis, Eq. (20) can be used to estimate the saturation length by finding the 
$z$-position for which $\left\langle b^{2}\right\rangle_{\text {shot }} \sim 1$ for the various positions within the electron beam.

\section{NUMERICAL RESULTS}

In our treatment so far, we have assumed that the current and nonlinear chirp profiles are arbitrary. For the purpose of numerical illustration, we now select a truncated parabolic current profile of the form $\chi(\theta)=1-\left(\theta-\theta_{m}\right)^{2} / \theta_{m}^{2}$ and a nonlinear chirp profile given by $Y(\theta)=\mu_{3}\left(\theta-\theta_{m}\right)^{3}$, where $\mu_{3}$ is a cubic chirp coefficient, these relations being valid for $0 \leq \theta \leq \theta_{b}=2 \theta_{m}$. The logic of this particular selection can be justified in the following way: since the chirp profile of the beam is actually shaped by space charge effects before the amplification process, it can be modeled by the $\theta$-derivative of the current profile, which can be approximated by a Gaussian [15]. Thus, we have

$$
\begin{aligned}
\eta & =-\mu\left(\theta-\theta_{m}\right)-\mu_{3}\left(\theta-\theta_{m}\right)^{3} \\
& \propto \frac{d}{d \theta} \exp \left(-\frac{\left(\theta-\theta_{m}\right)^{2}}{2 \sigma_{\theta}^{2}}\right),
\end{aligned}
$$

up to third order terms in $\theta-\theta_{m}$. This motivates the selection of a cubic chirp profile and also correlates $\mu_{3}$ with $\mu$ via the relation $\mu_{3}=-\mu /\left(2 \sigma_{\theta}^{2}\right)$. As far as $\sigma_{\theta}$ is concerned, we can either choose it in an ad-hoc way or derive it by matching the parabolic and Gaussian current profiles up to second order in $\theta-\theta_{m}$. Apart from justifying the use of a parabolic profile, this manipulation yields $\sigma_{\theta}=\theta_{m} / \sqrt{2}$. Though not entirely self-consistent, this strategy allows us to adequately model the space chargeinduced chirp while preserving some degree of analyticity as far as the Green's function is concerned. This is so because, for the case of parabolic current/cubic chirp, the $\xi$-integral in Eq. (14) can be determined analytically, a feature which enhances computational efficiency.

Additionally, it is essential to introduce an appropriate scaling in order to facilitate our numerical calculations. Specifically, the Green's function can be written as

$$
\begin{aligned}
G= & -\frac{\exp \left(i \Psi_{2}\right)}{2 \pi i} \int_{-\infty+i \hat{y}}^{+\infty+i \hat{y}} \frac{d \hat{\lambda}}{\hat{\lambda}} \exp \left(-i \hat{\lambda}\left[\bar{z}-\left(\hat{\theta}-\hat{\theta}_{j}\right)\right]\right. \\
& \left.-i \int_{\hat{\theta}_{j}}^{\hat{\theta}} \frac{d \hat{\xi} \hat{\chi}(\hat{\xi})}{\left[\hat{\lambda}+\hat{\Delta}_{0}\left(\hat{\xi}-\hat{\theta}_{j}\right)+\hat{Y}(\hat{\xi})-\hat{Y}\left(\hat{\theta}_{j}\right)\right]^{2}}\right)
\end{aligned}
$$

where the following dimensionless variables and constants have been introduced: $\hat{\lambda}=\lambda / \rho, \hat{\xi}=2 \rho \xi, \quad \bar{z}=2 \rho k_{u} z$, $\hat{\theta}=2 \rho \theta, \hat{\theta}_{j}=2 \rho \theta_{j}, \hat{\chi}(\hat{\xi})=1-\left(\hat{\xi}-\hat{\theta}_{m}\right)^{2} / \hat{\theta}_{m}^{2}$, and $\hat{Y}(\hat{\xi})=$ $\hat{\mu}_{3}\left(\hat{\xi}-\hat{\theta}_{m}\right)^{3}$, with $\hat{\theta}_{m}=2 \rho \theta_{m}, \hat{\mu}_{3}=\mu_{3} /\left(8 \rho^{4}\right)$, and $\hat{\Delta}_{0}=$ $\left(\mu-\bar{a}_{1}\right) /\left(2 \rho^{2}\right)=\hat{\mu}-\hat{a}_{1}$. The main advantage of this scaling is that the new dimensionless quantities are of the order of unity. This property can be utilized in order to elucidate and justify some of the approximations that where introduced in the theory section. Specifically, the gain length $l_{g}$ can be roughly estimated through the condition $\bar{z}=2 \rho k_{u} z \sim 1$, which yields $l_{g} \sim\left(2 \rho k_{u}\right)^{-1}=\lambda_{u} /(4 \pi \rho) \gg \lambda_{u}$. In a similar way, the cooperation length $l_{c}$ may be obtained from $\hat{\theta}=2 \rho \theta=2 \rho k_{r} z_{b} \sim 1$, i.e., $l_{c} \sim\left(2 \rho k_{r}\right)^{-1}=$ $\lambda_{r} /(4 \pi \rho) \gg \lambda_{r}$. We also recall that, in disregarding the $z$-dependence of the background distribution $f_{0}$, we made use of the approximation $\theta \gg 2 k_{u} \eta z$. Taking $\eta \sim \mu \theta$, this requirement is equivalent to $2 \mu k_{u} z \ll 1$ or $\rho \hat{\mu} \bar{z} \ll 1$ (valid since $\rho \ll 1$ ). Similar arguments may be invoked in order to validate the remaining assumptions.

From a practical point of view, the numerical calculation of the Green's function $G$ via the contour integral of Eq. (22) is facilitated by the stationary phase approximation. As a reminder, this technique approximates an integral of the form $\int d \hat{\lambda} P(\hat{\lambda}) \exp (i E(\hat{\lambda}))$ with the expression $P\left(\hat{\lambda}_{0}\right) \exp \left(i E\left(\hat{\lambda}_{0}\right)\right) \sqrt{2 \pi i / E^{\prime \prime}\left(\hat{\lambda}_{0}\right)}$, where $\hat{\lambda}_{0}$ is a stationary point of $E(\hat{\lambda})\left(E^{\prime}\left(\hat{\lambda}_{0}\right)=0\right)$ and $\left|E\left(\hat{\lambda}_{0}\right)\right| \gg 1$. In our case, this approximation is sufficiently accurate in the latter (or high-gain) stage of the linear regime. Finally, we note that, in terms of our scaling, Eqs. (17) and (20) become

$$
P_{r}(\hat{\theta}, \bar{z})=2 \gamma_{0} m c^{3} k_{r} \rho^{2} \int d \hat{\theta}_{j} \hat{\chi}\left(\hat{\theta}_{j}\right)\left|G\left(\hat{\theta}_{,} \hat{\theta}_{j}, \bar{z}\right)\right|^{2}
$$

and

$\left\langle b^{2}(\hat{\theta}, \bar{z})\right\rangle_{\text {shot }}=\frac{4 \pi \rho}{n_{0} \lambda_{r}} \int d \hat{\theta}_{j} \hat{\chi}\left(\hat{\theta}_{j}\right)\left|\hat{G}_{b}\left(\hat{\theta}, \hat{\theta}_{j}, \bar{z}\right)\right|^{2} / \hat{\chi}^{2}(\hat{\theta})$,

respectively, where $\hat{G}_{b}=(\partial / \partial \bar{z}+\partial / \partial \hat{\theta}) G\left(\hat{G}_{b}\right.$ is calculated through numerical differentiation of $G$ ). Along with Eq. (22), these relations complete the array of tools necessary for carrying out the numerical study presented below.

\section{A. Comparison with 1D simulation}

To start with, we select a parameter set that roughly approximates a plausible configuration of the X-LEAP eSASE experiment at SLAC [16]. This involves the generation of $800 \mathrm{eV}$ photons $\left(\lambda_{r}=1.55 \mathrm{~nm}\right)$ with a standard LCLS undulator $\left(\lambda_{u}=3 \mathrm{~cm}, K_{0}=3.5\right)$ and a $4.24 \mathrm{GeV}$ beam with a peak current of $4.5 \mathrm{kA}$. The average beta function $\beta_{x}$ is about $10 \mathrm{~m}$, which corresponds to a rms beam size $\sigma_{x}=\sqrt{\epsilon_{x} \beta_{x}} \approx 25 \mu \mathrm{m}$ (for a transverse normalized emittance $\gamma_{0} \epsilon_{x} \approx 0.5 \mu \mathrm{m}$ ). The $\rho$ parameter is about $2 \times 10^{-3}$, while we also assume zero uncorrelated energy spread. The latter is not actually true in the experimental setup but this discrepancy is balanced by choosing a lower peak current for the beam ( $4.5 \mathrm{kA}$ instead of 6-7 kA). As far as the other parameters are concerned, we take $z=$ $11.45 \mathrm{~m}$ (corresponding to $\bar{z}=9.2$ ) and consider two different cases for the bunch length: case A with a bunch 


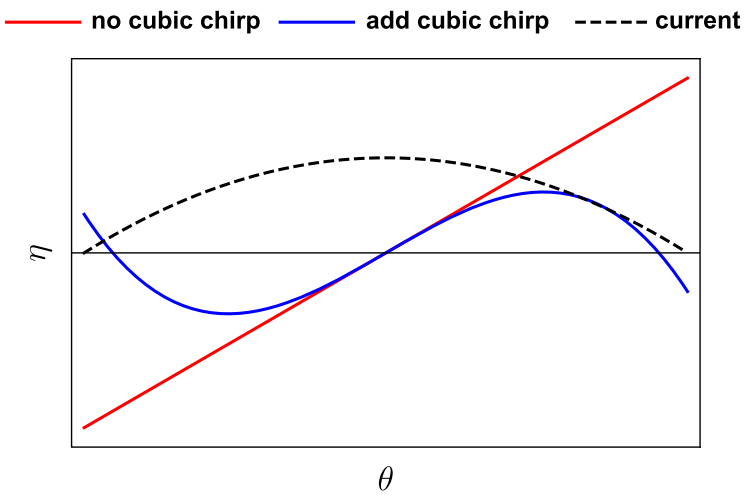

FIG. 1. Generic schematic that illustrates the chirp profile of the electron beam vs the current profile.

length of $500 \mathrm{~nm}\left(\hat{\theta}_{b}=7.8\right.$, so that $\left.\hat{\theta}_{b}<\bar{z}\right)$ and case B with a longer bunch length of $750 \mathrm{~nm}$ (for which $\hat{\theta}_{b}=11.7$ and $\left.\hat{\theta}_{b}>\bar{z}\right)$. For both of these cases, $\mu$ is given by $\mu=-\left(\Delta \gamma / \gamma_{0}\right) / \theta_{b}$, where $\Delta \gamma=\Delta E / m c^{2}$ and $\Delta E=$ $30 \mathrm{MeV}$ is the total energy variation due to the linear chirp. Moreover, we choose $\sigma=\sigma_{\theta} / k_{r}=160 \mathrm{~nm}$. This leads to a negative linear chirp, which is compensated by the appropriate (reverse) taper, leaving only the cubic component contribution (see the schematic of Fig. 1).

In Figs. 2 and 3, we plot the (shot-averaged) radiation power and bunching factor (the latter defined as $\sqrt{\left\langle b^{2}\right\rangle_{\text {shot }}}$ ) as functions of the position $z_{b}$ along the bunch. The theoretical values are calculated by Eqs. (23) and (24) while the simulation values are obtained from a 1D FEL simulation code (some details for the latter are given in [9]). Reasonable agreement is observed between the two approaches, which enables us to build confidence in our analysis.

\section{B. Parameter study}

Having verified our formalism, we use it in a simple parameter study that is relevant for an eSASE-based FEL configuration. In particular, we scan the electron pulse duration $t_{e}=l_{b} / c=\theta_{b} /\left(c k_{r}\right)$ while keeping constant both the peak current $I_{p}$ and the product $\mu \times t_{e}$. According to our previous discussion, the latter is proportional to the total energy variation $\Delta E$, which is kept fixed at $35 \mathrm{MeV}$. Using the Green's function method, we obtain the shot-averaged power profiles for the pure, matched linear chirp case (red data in Figs. 4-6) and for the case with the added nonlinear chirp component (blue data in the same figures). This particular scan was performed assuming $\mu_{3}=-\mu /\left(2 \sigma_{\theta}^{2}\right)$ and $\sigma_{\theta}=\theta_{m} / \sqrt{2}$. In Fig. 4 , we plot the two contrasting power profiles for a bunch length of $1.5 \mathrm{fs}$. A power suppression due to the addition of the cubic chirp is immediately evident. Moreover, we find that the radiation full-width-at-half-maximum (FWHM) is also reduced. These observations are confirmed in Fig. 5, where the

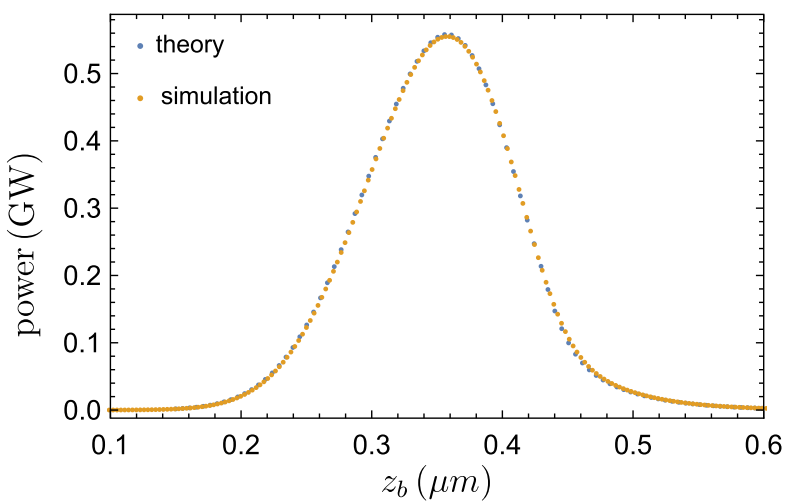

(a) radiation power

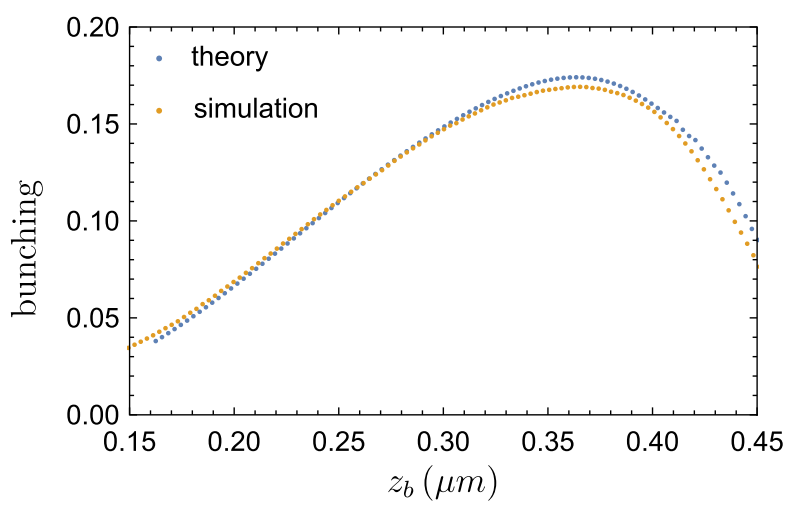

(b) bunching factor

FIG. 2. Shot-averaged radiation power and bunching factor along the e-beam (case A: $z=11.45 \mathrm{~m}, 0.50 \mu \mathrm{m}$ bunch length, $\sigma=\sigma_{\theta} / k_{r}=160 \mathrm{~nm}, \Delta E=30 \mathrm{MeV}, 400$ shot average, matched linear chirp). The blue/brown curves represent theory/simulation data.

peak radiation power and the FWHM are plotted as functions of $t_{e}$. A marked linear dependence of the FWHM with $t_{e}$ is observed, along with what appears to be a constant vertical shift (reduction) due to the cubic chirp. Moreover, Fig. 6 verifies that, even for the same peak power level, the radiation pulse length is reduced when the nonlinear chirp is added. This effect can probably be attributed to the reduction of the effective electron bunch length due to the introduction of a chirp component that is not compensated by the undulator taper.

The observed linear character of the FWHM plot (red curve) can be reproduced in a heuristic fashion through the following argument: since the $\rho$ parameter scales according to $\rho \sim n_{0}^{1 / 3} \sim I_{p}^{1 / 3}$, we can define a $\theta$-dependent, effective FEL parameter $\rho_{\text {eff }}=\rho(\chi(\theta))^{1 / 3}$ by plugging in the scaled current profile $\chi(\theta)=1-\left(\theta-\theta_{m}\right)^{2} / \theta_{m}^{2}$ [we recall that $\left.I=I_{p} \chi(\theta)\right]$. Combining this with the power growth relation $P_{r}=P_{0} \exp \left(z / L_{g}\right)$, where $L_{g} \sim \rho_{\text {eff }}^{-1}$ is the local power gain length, we find a Gaussian power profile of the form $\exp \left[-\left(z / L_{0}\right)\left(\theta-\theta_{m}\right)^{2} /\left(3 \theta_{m}^{2}\right)\right]$, where $L_{0}=\lambda_{u} /(4 \pi \sqrt{3} \rho)$ is the basic (minimum) value for the power gain length (which is about $0.70 \mathrm{~m}$ for our parameters). This predicts 


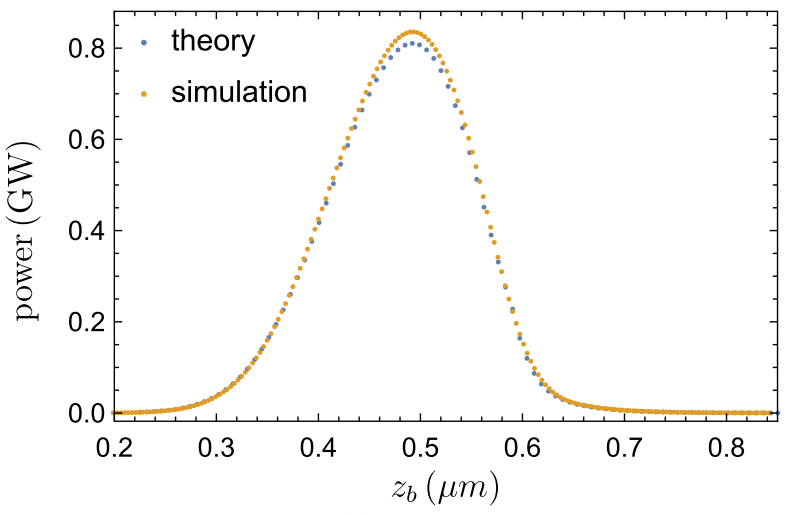

(a) radiation power

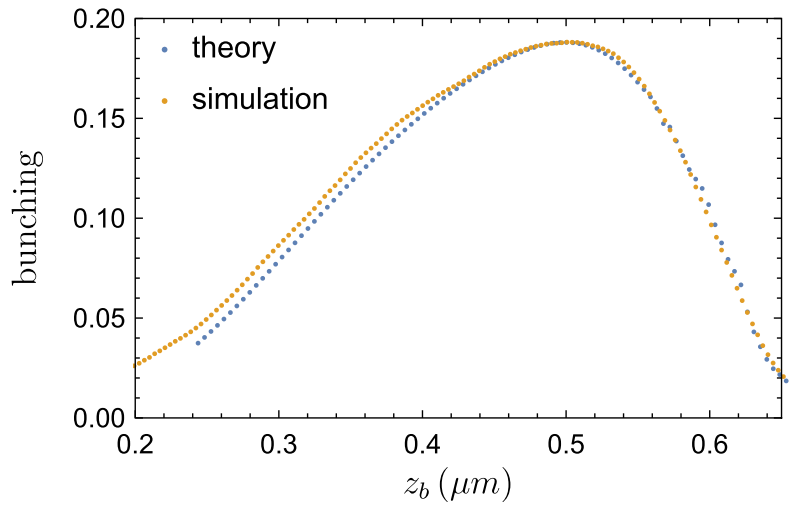

(b) bunching factor

FIG. 3. Shot-averaged radiation power and bunching factor along the e-beam (case B: $z=11.45 \mathrm{~m}, 0.75 \mu \mathrm{m}$ bunch length, $\sigma=\sigma_{\theta} / k_{r}=160 \mathrm{~nm}, \Delta E=30 \mathrm{MeV}, 400$ shot average, matched linear chirp). The blue/brown curves represent theory/ simulation data.

maximization of the radiation power in the middle of the electron bunch, which is not very accurate (in fact, it happens closer to the head of the beam, see Fig. 4). On the other hand, the FWHM is simply $t_{e} \times \sqrt{3 \log 2 /\left(z / L_{0}\right)}$, a

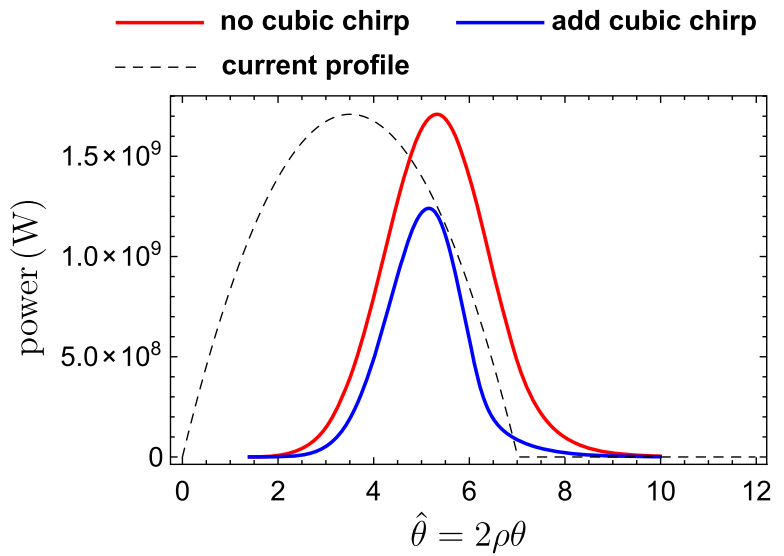

FIG. 4. Shot-averaged radiation power profiles for a matched linear chirp with or without the cubic chirp component (blue and red curves, respectively). The bunch length is $1.5 \mathrm{fs}$ and we assume $z=12.3 \mathrm{~m}$. The dashed line denotes the scaled current profile.

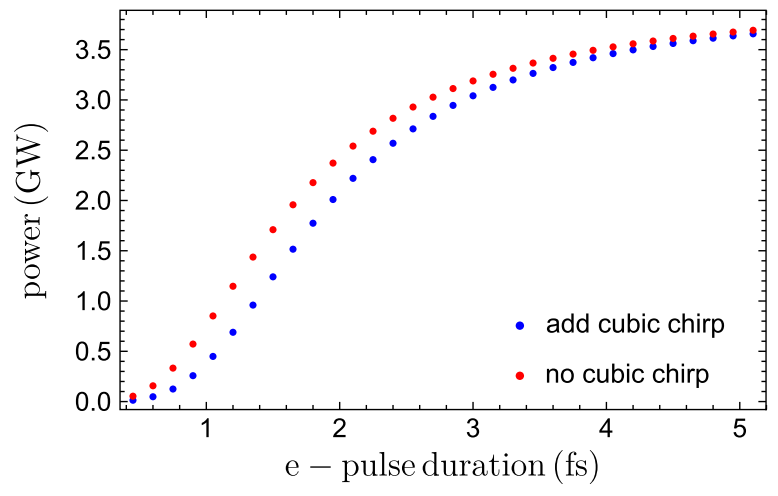

(a) peak radiation power

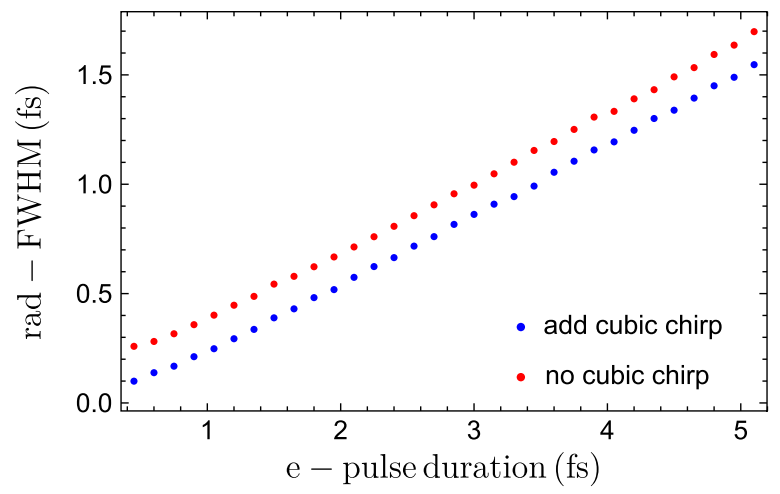

(b) radiation FWHM

FIG. 5. Radiation peak power and FWHM vs the electron bunch length (same color convention as in Fig. 4).

result which exhibits linear dependence with respect to $t_{e}$ with a slope of about 0.35 . This is very close to the value calculated from the Green's function data (0.32), even though the simple model under study does not take into account detuning effects etc. In any event, the scaling predicted by the above formula may be of interest when doing back-of-the-envelope calculations involving attosecond-style pulses.

We would also like to point out that, besides elucidating the underlying physics, the Green's function formalism

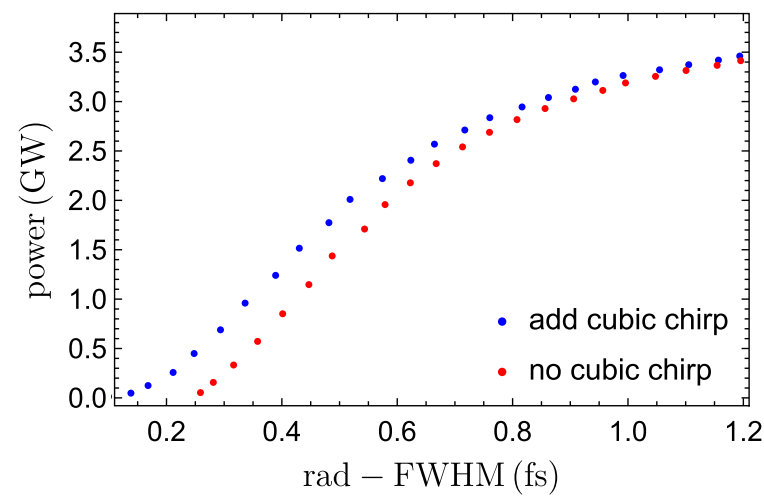

FIG. 6. Radiation peak power vs FWHM (using the data of Fig. 5). 
allows us to get a sense of what the optimum setup of the FEL configuration is. Apart from determining the proper matching strategy involving the linear chirp and taper, we can study the interplay between power suppression and FWHM reduction due to nonlinear chirp in order to find a suitable working point, even for realistic, machine-relevant scenarios. Moreover, even though our theoretical results are based on a one-dimensional model, the essential conclusions remain valid in a more complicated (3D) setup. To demonstrate this, in Figs. 7 and 8, we show the results of a GENESIS-based [17] 3D FEL simulation involving a chirped drive beam. Specifically, Fig. 7 shows the chirp profiles for the two cases considered (i.e., pure linear chirp and linear chirp plus cubic component) while Fig. 8 presents the output of the FEL simulation in terms of

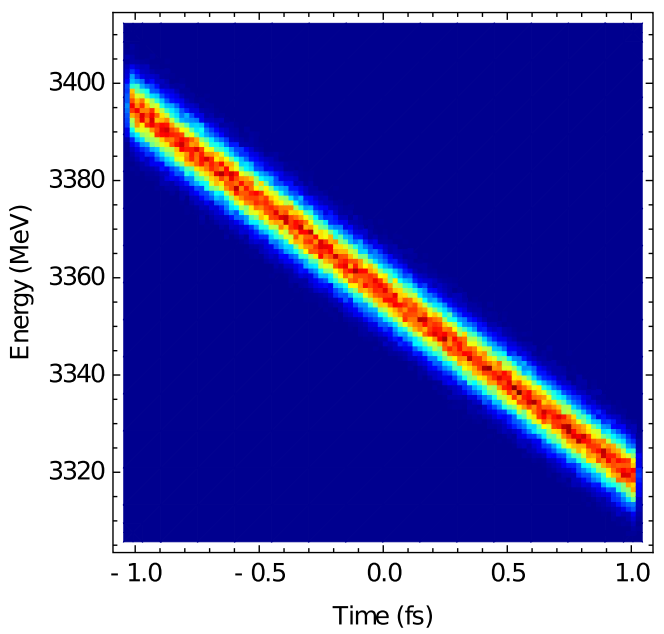

(a) pure linear chirp

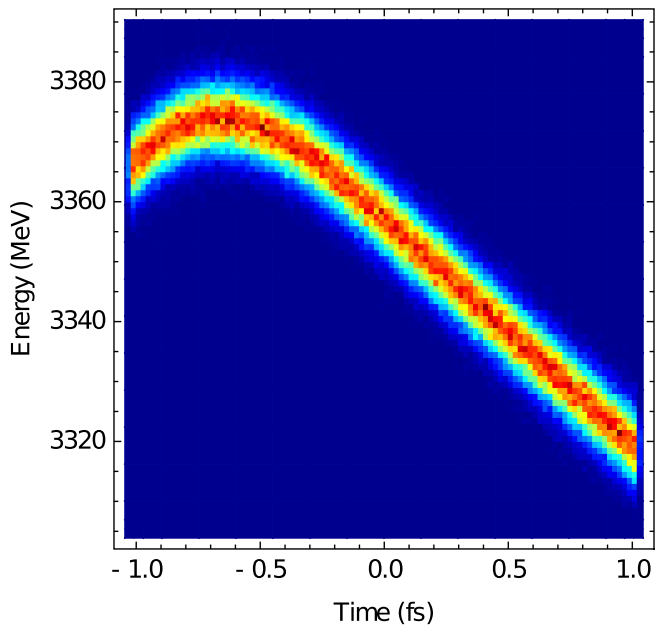

(b) adding a cubic chirp component

FIG. 7. Chirp profiles of the electron beam used in GENESIS simulations. The upper graph shows the case of a pure linear chirp, while the lower figure shows the phase-space profile after the addition of a cubic chirp component at the head of the beam (left-hand side of the graph). This example is pretty close to the matched case in terms of linear chirp-taper compensation.

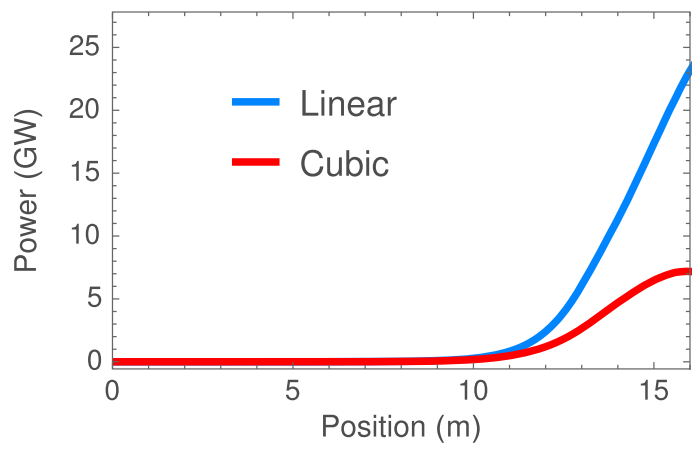

(a) radiation power

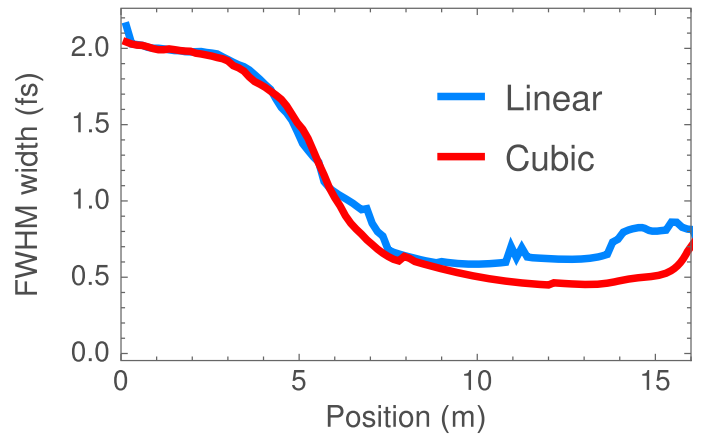

(b) bunching factor

FIG. 8. Shot-averaged radiation power and bunching factor along the undulator for the drive beam of Fig. 7 (the average involves ten SASE shots). A power reduction/pulse shortening effect due to nonlinear chirp is evident in the high-gain stage of the linear region. The output radiation FWHM is about a third of the bunch length of the electron beam.

the radiation power and bunching factor along the undulator. The simulation parameters we used are as follows: $500 \mathrm{eV}$ photon energy, LCLS-like initial undulator parameter $\left(K_{0}=3.5\right.$, increasing linearly to 3.85 over the undulator length of $27 \mathrm{~m}$ ), flattop current profile with a $2 \mathrm{fs}$ bunch length and a $4.5 \mathrm{kA}$ current and $0.4 \mu \mathrm{m}$ transverse normalized emittance. The beam is considered matched to a standard LCLS lattice, which translates into a roughly constant transverse beam size $\sim 20 \mu \mathrm{m}$. As in the case of 1D theory, Fig. 8 shows that adding a nonlinear (cubic) chirp to the longitudinal profile of the beam clearly suppresses the output power in the presaturation region but also reduces the effective pulse length of the radiation.

\section{CONCLUSIONS}

We have developed a one-dimensional, time-dependent theoretical formalism that can adequately model the amplification portion of an eSASE-based FEL configuration. Our formalism includes startup from noise, radiation slippage, short-pulse effects, electron beam chirp (linear and nonlinear) and linear undulator taper. Working in the context of a self-consistent, linearized Maxwell-Vlasov analysis, we derived a Green's function for the amplitude of the FEL 
radiation, which allows us to determine the basic properties of the radiation pulse prior to the onset of saturation. As part of our derivation, we provide a rigorous proof of the well-known compensation condition between linear chirp and taper and generalize previous analytical results for the Green's function. Reasonable agreement is observed between our formalism and the output of a 1D FEL simulation code, while our technique is robust enough to enable us to perform fast parameter scans. In the course of such a parameter study, we explored the variation of the radiation FWHM with respect to the electron bunch length (establishing a linear dependence between the two) and also observed an interesting pulse-shortening effect due to the presence of a nonlinear chirp component in the e-beam. This effect may have some practical applications in terms of offering a scheme for further pushing the attainable radiation pulse length well into the attosecond region.

\section{ACKNOWLEDGMENTS}

We would like to thank J. MacArthur for useful discussions and assistance with 1D FEL simulations. This work was supported by the U.S. Department of Energy, Office of Science, under Contract No. DEAC02-76SF00515. A. M.'s work was supported by the Department of Energy's BES-ADR program under FWP 100317.

[1] W. Ackermann et al., Operation of a free-electron laser from the extreme ultraviolet to the water window, Nat. Photonics 1, 336 (2007).

[2] P. Emma et al., First lasing and operation of an angstromwavelength free-electron laser, Nat. Photonics 4, 641 (2010).

[3] T. Ishikawa et al., A compact x-ray free-electron laser emitting in the sub-ngstrm region, Nat. Photonics 6, 540 (2012).

[4] H.S. Kang et al., Hard X-ray free-electron laser with femtosecond-scale timing jitter, Nat. Photonics 11, 708 (2017).
[5] A. Zholents, Method of an enhanced self-amplified spontaneous emission for x-ray free electron lasers, Phys. Rev. ST Accel. Beams 8, 040701 (2005).

[6] E. L. Saldin, E. A. Schneidmiller, and M. V. Yurkov, Selfamplified spontaneous emission FEL with energy-chirped electron beam and its application for generation of attosecond x-ray pulses, Phys. Rev. ST Accel. Beams 9, 050702 (2006).

[7] Z. Huang and K. J. Kim, Review of x-ray free-electron laser theory, Phys. Rev. ST Accel. Beams 10, 034801 (2007).

[8] S. Krinsky, Transient analysis of free-electron lasers with discrete radiators, Phys. Rev. E 59, 1171 (1999).

[9] Z. Huang and K. J. Kim, Effects of bunch density gradient in high-gain free-electron lasers, Nucl. Instrum. Methods Phys. Res., Sect. A 445, 105 (2000).

[10] S. Krinsky and Z. Huang, Frequency chirped self-amplified spontaneous-emission free-electron lasers, Phys. Rev. ST Accel. Beams 6, 050702 (2003).

[11] C. B. Schroeder, C. Pellegrini, S. Reiche, J. Arthur, and P. Emma, Chirped-beam two-stage free-electron laser for high-power femtosecond x-ray pulse generation, J. Opt. Soc. Am. B 19, 1782 (2002).

[12] A. A. Lutman, G. Penco, P. Craievich, and J. Wu, Impact of an initial energy chirp and an initial energy curvature on a seeded free electron laser: The Green's function, J. Phys. A 42, 045202 (2009).

[13] Z. Huang and G. Stupakov, Free electron lasers with slowly varying beam and undulator parameters, Phys. Rev. ST Accel. Beams 8, 040702 (2005).

[14] L. Giannessi et al., Self-Amplified Spontaneous Emission Free-Electron Laser with an Energy-Chirped Electron Beam and Undulator Tapering, Phys. Rev. Lett. 106, 144801 (2011).

[15] Y. Ding, Z. Huang, D. Ratner, P. Bucksbaum, and H. Merdji, Self-amplified spontaneous emission free-electron laser with an energy-chirped electron beam and undulator tapering, Phys. Rev. ST Accel. Beams 12, 060703 (2009).

[16] J. P. MacArthur, J. P. Duris, Z. Huang, and A. Marinelli, High power sub-femtosecond X-ray pulse study for the LCLS, Proceedings of the 8th International Particle Accelerator Conference, Copenhagen, Denmark, 2017, http://jacow.org/ipac2017/papers/wepab118.pdf.

[17] S. Reiche, GENESIS 1.3: A fully 3D time-dependent FEL simulation code, Nucl. Instrum. Methods Phys. Res., Sect. A 429, 243 (1999). 